\title{
Acute effects of flavonoid-rich blueberry on cognitive and vascular function in healthy older adults
}

\author{
Georgina F. Dodd ${ }^{\mathrm{a}, \mathrm{b}}$, Claire M. Williams ${ }^{\mathrm{b}}$, Laurie T. Butler ${ }^{\mathrm{b}}$ and Jeremy P. E. Spencer ${ }^{\mathrm{a}, *}$ \\ ${ }^{a}$ Department of Food and Nutritional Sciences, Hugh Sinclair Unit of Human Nutrition, School of Chemistry, \\ Food and Pharmacy, University of Reading, Berkshire, UK \\ ${ }^{\mathrm{b}}$ Department of Psychology, School of Psychology and Clinical Language Sciences, University of Reading, \\ Berkshire, UK
}

\begin{abstract}
.
BACKGROUND: Consumption of flavonoids, natural compounds found in foods such as berries and cocoa, have been shown to be beneficial for cognitive function. However, less is known about potential mechanisms and acute benefits for the older population.

OBJECTIVE: To determine whether acute intake of flavonoid-rich blueberry could have beneficial effects on cognitive function in a sample of healthy older adults and identify possible mechanisms.

METHODS: A cross-over randomised controlled trial $(\mathrm{RCT})$ was conducted $(\mathrm{N}=18)$ with volunteers receiving a flavonoidrich blueberry beverage (579mg of antho- and pro-cyanidins) on one visit and a sugar-matched control on another. Cognitive function was measured at baseline, 2 and 5 hours post consumption of the intervention and blood pressure (BP), arterial stiffness and plasma brain-derived neurotrophic factor (BDNF) concentration at baseline and an hour post-intervention.

RESULTS: Whilst there was no significant effect of the intervention on global cognitive function, performance was significantly different at 2 compared to 5 hours following the control beverage $(p<0.05)$, with a decline in performance relative to baseline at 2 hours, whereas cognitive function improved following the blueberry beverage at both post intervention time points. There was also a trend towards the blueberry beverage attenuating the increase in systolic BP evident following the control drink $(p=0.08)$. Finally, there was a decrease in plasma concentration of BDNF post consumption of the control drink which was attenuated following the blueberry beverage, although not significantly $(p>0.05)$.

CONCLUSIONS: A single dose of flavonoid-rich blueberry could have a potentially protective effect on cognitive function in healthy older adults, possibly due to improved cerebrovascular function and positive interactions with cell signalling pathways involved in cognitive processes.
\end{abstract}

Keywords: Blueberry plants, flavonoids, anthocyanins, healthy aging, cognition, cognitive function, vascular endothelium, cerebrovascular circulation

\section{Introduction}

Maintaining cognitive health as we age is a growing concern, with significant research effort focussed on preventing cognitive decline and delaying the onset of neurodegenerative diseases such

\footnotetext{
${ }^{*}$ Corresponding author: Professor Jeremy P. E. Spencer; Address: Hugh Sinclair Unit of Human Nutrition, Department of Food \& Nutritional Sciences, School of Chemistry, Food \& Pharmacy, PO Box 226, Harry Nursten Building, Whiteknights, University of Reading, Reading, Berkshire, RG6 6AP. UK. Tel.: +44 (0) 118378 8724; Fax: +44 0118 931; E-mail: j.p.e.spencer@reading.ac.uk.
}

as Alzheimer's and Parkinson's Disease. Diet and lifestyle factors could play a significant role in maintaining cognitive function with increasing age. Recently, interest has increased in the role of flavonoids, naturally occurring plant compounds, which are found in most fruit and vegetables, as there is a growing body of evidence suggesting that supplementation with flavonoid-rich foods could be beneficial for cognitive function [1-3] and may potentially promote healthy cognitive ageing.

Acute supplementation with flavanol-rich cocoa was found to be beneficial during tasks with high cognitive demand [4]. In addition, consumption of 
flavanone-rich orange juice has been shown to be beneficial following both acute [5] and chronic [6] intake in healthy adults. Berries are also known to be a rich source of flavonoids and recent research by Nilsson et al. [7] in healthy adults found that consumption of a mixed berry beverage for five weeks resulted in improved working memory performance, in addition to beneficial effects on various cardiometabolic parameters, compared to a control beverage. However, this was in combination with fibre and therefore effects cannot be attributed to flavonoids alone. Also, no baseline measure of cognitive function was taken and it cannot be ascertained whether performance increased as a result of the intervention. Furthermore, evidence of acute effects of berry-derived flavonoids on cognition comes from recent research by Watson et al. suggesting that acute supplementation with blackcurrant can lead to benefits in terms of brain function which may be due to inhibition of monoamine oxidase-B [8]. In addition, an acute study with healthy younger adults found evidence of improved cognitive function and mood following consumption of purple grape juice, including reduced reaction time on a composite measure of attention and increased self-reported calmness [9].

Blueberries are one of the richest sources of anthocyanins in comparison with other berries [10] and therefore have also been of interest with regards to potential cognitive benefits. Acute intervention trials in children have shown beneficial effects of blueberry supplementation in terms of improved delayed word recall performance (an aspect of episodic memory), which is thought to be due to more efficient encoding [11]. It has also been postulated that benefits are more likely to occur for tasks requiring greater cognitive demand (i.e. more challenging tasks requiring greater cognitive resource) and that effects follow a dose-response pattern [12].

Proposed mechanisms of action through which flavonoids may have beneficial effects on cognitive performance include protection against neuroinflammation [13, 14], cerebrovascular effects leading to improved cerebral blood flow or volume [15-18], and their positive impact on cell signalling pathways promoting neurogenesis, synaptic plasticity and expression of proteins involved with memory and learning such as brain-derived neurotrophic factor (BDNF) [19]. Recent evidence in support of the latter comes from a study by Rendeiro et al. who showed that healthy younger rats fed a blueberry diet demonstrated improved spatial memory performance which was thought to be due to the interaction of flavonoids with the ERK-CREB-BDNF pathway [20]. Notwithstanding the few studies which have proposed alternative mechanisms such as modulation of glucose regulation [21], effects on insulin resistance [22] and inhibition of monoamine oxidase-B [8], research investigating potential mechanisms of action in vivo is lacking.

Despite the potential for flavonoid-rich foods to be beneficial for cognitive health, human research into the effects of flavonoid supplementation in an ageing population, particularly with regard to anthocyaninrich foods such as blueberries, is limited. Cognitive performance is known to decline over the lifespan and therefore this population may benefit most from early dietary intervention to delay impairment. As such, it is critical to determine the nature of any benefit from flavonoid supplementation in this population both acutely as well as chronically. Indeed, Krikorian and colleagues found that in a group of individuals with Mild Cognitive Impairment, supplementation with Concord grape juice for 12 weeks improved item acquisition on the California Verbal Learning Test, a memory measure assessing verbal learning and retention, compared to placebo. There were also trends towards improved delayed verbal recall and spatial memory [23].

In addition, following on from their previous study, the same authors later reported that 12 weeks of daily supplementation with wild blueberry juice (approximately 400-600mg anthocyanins depending on body weight) resulted in improved performance on a verbal paired associate learning task compared to the placebo group when controlling for baseline performance. There was also a positive effect on mood, with a trend towards reduced depressive symptoms. Furthermore, blueberry supplementation resulted in a trend towards reduced glucose levels which suggests that modulating glucose metabolism may be a mechanism through which blueberry-derived flavonoids could be beneficial for cognitive function [21]. However, the sample was small (Blueberry group: $\mathrm{N}=9$; Placebo group: $\mathrm{N}=7$ ) and the placebo was designed to match a grape intervention and therefore was not optimally matched to the blueberry beverage. The absence of an adequate placebo makes it difficult to draw conclusions as to the efficacy of the blueberry intervention. Nevertheless, with the use of comparable methodology, it was established that effects were unlikely to be due to practice.

Although such earlier research was promising, studies in this population determining the efficacy of a single dose of an intervention which has been charac- 
Table 1

Demographic characteristics

\begin{tabular}{|c|c|c|c|}
\hline & M & SD & Range \\
\hline Age, y & 68.72 & 3.30 & $62-73$ \\
\hline MMSE score $(/ 30)^{a}$ & 28.41 & 1.42 & $26-30$ \\
\hline BSI depression score $(/ 24)^{a}$ & 59 & 62 & $0-2$ \\
\hline HADS anxiety score $(/ 21)^{b}$ & 4.28 & 3.79 & $0-16$ \\
\hline Education, y & 14.89 & 3.71 & $10-24$ \\
\hline Crystallised IQ - NART score $(/ 50)^{b, c}$ & 40.00 & 7.08 & $28-49$ \\
\hline Fluid IQ - Cattell's percentile rank ${ }^{a}$ & 50.06 & 29.75 & $3-99$ \\
\hline Systolic BP, mmHg & 135.02 & 7.34 & $105.67-156.67$ \\
\hline Diastolic BP, $\mathrm{mmHg}$ & 78.55 & 3.38 & $58.00-94.00$ \\
\hline BMI, $\mathrm{kg} / \mathrm{m}^{2}$ & 25.89 & 4.46 & $21.12-35.08$ \\
\hline Fruit consumption (portions per day) & 2.53 & 1.47 & $1-6$ \\
\hline Vegetable consumption (portions per day) & 2.58 & .90 & $1-5$ \\
\hline
\end{tabular}

${ }^{a}$ Data was missing for one volunteer, ${ }^{b}$ Data for two volunteers was missing, ${ }^{c}$ data for three older adult volunteers was acquired from an existing database. MMSE, Mini Mental State Examination; BSI, Brief Symptom Inventory; HADS, Hospital Anxiety and Depression Scale; NART, National Adult Reading Test; BMI, Body Mass Index.

terised with regards to flavonoid content, whilst also aiming to establish potential mechanisms of action in vivo, are currently lacking. The aim of the current research was therefore to determine whether an acute dose of flavonoid-rich blueberry in the form of a beverage would lead to improved global cognitive function in healthy older adults. Also, whether there would be improvements in particular cognitive abilities as measured by individual tasks, similar to those found to be sensitive to chronic blueberry supplementation in previous studies, for example, episodic memory [24, 25], which is the ability to remember experiences and specific events. Parameters related to vascular health and plasma concentration of BDNF were also measured in an attempt to identify potential mechanisms underlying any beneficial cognitive effects.

\section{Materials and methods}

\subsection{Participants}

Eighteen fluent English speakers (10 women; 8 men) aged 60-75 years (mean+/- SD age: $68.7+/$ $3.3 \mathrm{y}$ ), were recruited from the local area (Table 1). Volunteers with a Mini Mental State Examination (MMSE) score of $\leq 25$, a depression index score of $\geq 11$ on the Brief Symptom Inventory (BSI), both assessed during screening, and those taking medication for hypertension were excluded. Based on an alpha of $0.05,80 \%$ power and a Cohen's $d$ effect size of 0.8 , a $G^{*}$ power [26] calculation determined that a total sample size of 18 would be required ${ }^{1}$. In addition, considering the cross-over design of the study and that previous acute flavonoid intervention trials of similar design have shown positive effects on cognitive performance with a comparable number or fewer subjects [27, 28], a sample size of 18 for this trial was considered appropriate. All participants completed both arms of the trial.

\subsection{Interventions}

The intervention beverages were comprised of powdered sachets homogenised with $300 \mathrm{~mL}$ of semi-skimmed milk. Refrigerated fresh highbush blueberries were washed, dried, blended, frozen, freeze-dried and milled to create the blueberry powder (approximately 30g per drink) which contained a combined total of $579 \mathrm{mg}$ of antho- and pro-cyanidins (508mg and $71 \mathrm{mg}$ respectively) determined using HPLC (Table 2). The dosage was equivalent to approximately $200 \mathrm{~g}$ of fresh blueberries which is achievable in the context of the habitual diet, as $160 \mathrm{~g}$ of blueberries is equivalent to two UK servings [29]. Also, the total dose of antho- and pro-cyanidins was comparable to previous studies in the literature that have shown acute effects of berry-derived flavonoids on cognitive performance [8]. The control powder was formulated to match the blueberry drink (Table 3) for compounds that could potentially affect cognitive

\footnotetext{
${ }^{1}$ This was based on two groups and three repeated measurements as this appeared to be the most appropriate approach given that $G^{*}$ Power does not facilitate convenient calculations for two factor repeated measures designs.
} 
Table 2

Antho- and pro-cyanidin content of the control and blueberry interventions

\begin{tabular}{lcc}
\hline & $\begin{array}{c}\text { Control } \\
\text { (per 19.9g } \\
\text { of powder) }\end{array}$ & $\begin{array}{c}\text { Blueberry } \\
\text { (per 30.1g } \\
\text { of powder) }\end{array}$ \\
\hline $\begin{array}{l}\text { Anthocyanidins (mg) } \\
\text { Delphinidin }\end{array}$ & 0 & 155.92 \\
Cyanidin & 0 & 24.98 \\
Petunidin & 0 & 86.09 \\
Peonidin & 0 & 3.91 \\
Malvidin & 0 & 236.89 \\
Total (mg) & 0 & $\mathbf{5 0 7 . 7 9}$ \\
Epicatechin oligomers (mg) & & \\
Monomer & 0 & 27.69 \\
Dimer & 0 & 18.36 \\
$\quad$ Trimer - Decamer & 0 & 24.98 \\
Total (mg) & 0 & $\mathbf{7 1 . 0 3}$ \\
Combined total (mg) & 0 & $\mathbf{5 7 8 . 8 2}$ \\
\hline
\end{tabular}

Table 3

Nutrient composition of the control powder and typical composition of raw blueberry

\begin{tabular}{lcc}
\hline Ingredient & $\begin{array}{c}\text { Quantity } \\
\text { per control } \\
\text { drink }\end{array}$ & $\begin{array}{c}\text { Quantity } \\
\text { per 200g of } \\
\text { raw blueberry }\end{array}$ \\
\hline Energy (kcal) & $75^{b}$ & 114 \\
Energy (kJ) & $319^{b}$ & 480 \\
Protein (g) & 0 & 1.48 \\
Total lipid (fat) (g) & 0 & 0.66 \\
Total sugars $(\mathrm{g}):$ & 19.92 & 19.92 \\
Fructose $(\mathrm{g})$ & 9.94 & 9.94 \\
Glucose $(\mathrm{g})$ & 9.76 & 9.76 \\
Sucrose $(\mathrm{g})$ & 0.22 & 0.22 \\
Lactose $(\mathrm{g})$ & 0 & 0 \\
Maltose $(\mathrm{g})$ & 0 & 0 \\
Galactose $(\mathrm{g})$ & 0 & 0 \\
Starch $(\mathrm{g})$ & 0 & 0.06 \\
Folate, total $(\mu \mathrm{g})$ & 0 & 12 \\
Vitamin C, total ascorbic acid $(\mathrm{mg})$ & 19 & 19.4 \\
Citric acid $($ added $)(\mathrm{g})$ & 1 & 0 \\
\hline
\end{tabular}

${ }^{a}$ Figures sourced from the USDA Nutrient database. ${ }^{b}$ Calculated according to total sugar content.

function, namely sugars and vitamin $\mathrm{C}$ content. Taste was also matched with the addition of $1 \mathrm{~g}$ of citric acid. Drinks were provided in an opaque cup complete with lid and black straw to mask the appearance of the contents so that the volunteers would remain blinded for the duration of the study. The sachets of powder for both the blueberry and the control intervention were stored in a $-20^{\circ} \mathrm{C}$ freezer and all drinks were made fresh on the day of testing as required and consumed immediately.

\subsection{Design}

A randomised, controlled, cross-over, intervention trial was conducted, with two drink conditions (blueberry beverage vs. control beverage) with global cognitive function as the primary endpoint. Cognitive performance was tested at baseline as well as at 2 and 5 hours post consumption of the intervention using matched versions of each of the cognitive tasks within the test battery which were comparable in terms of task difficulty. Blood pressure, stiffness index and a blood sample were taken at baseline and $60 \mathrm{~min}$ utes after the intervention to coincide with the known peak plasma concentration of metabolites following supplementation with anthocyanin-rich interventions [30]. Cognitive practice effects were expected during the course of the study, but drink order (whether blueberry or control drink was consumed on the first visit) was counterbalanced across the sample and change in performance following blueberry supplementation was compared with that following the control beverage so that any effects could be attributed to the blueberry intervention rather than practice.

\subsection{Procedure}

Volunteers were invited to the Hugh Sinclair Unit of Human Nutrition for a screening and familiarisation visit. They were asked to arrive fasted and once preliminary eligibility had been checked and informed consent procedures completed, measurements of height, weight and BP were taken. This was followed by a blood sample via venepuncture. During a light breakfast of toast and preserves with a tea/coffee and water, the volunteers completed several questionnaires. The BSI [31] was completed in order to exclude volunteers who may be depressed. Other questionnaires were issued to establish demographic, medical, and lifestyle information, as well as habitual anxiety levels and physical capability. The MMSE [32] was then administered as well as the Cattell's Culture Fair test of ' $\mathrm{g}$ ' [33] and the National Adult Reading Test [34] to assess fluid and crystallised intelligence respectively. The tasks measuring executive function were the most difficult within the cognitive test battery as they are more reliant on complex cognitive processes and therefore familiarisation with them prior to the visit was required. This also helped to minimise practice effects. Study days were arranged and volunteers were given standardised written instructions asking 
them to avoid alcohol and to adhere to a low polyphenol diet for the 24 hours prior to each study day. Specifically, they were asked not to consume foods and beverages rich in polyphenols such as fruit, vegetables, tea and cocoa. The breakfast and lunch provided during the study days also complied with the low-polyphenol diet and were the same for each visit. This was to ensure a low-polyphenol baseline for each study day and that the intervention was the only source of flavonoids during the visit.

On each test day, volunteers arrived fasted for each study day and adherence to the dietary restrictions was checked. Blood pressure was measured and a fasted blood sample taken via venepuncture. This was followed by the digital volume pulse (DVP) measurements. The volunteer was then given the standardised study breakfast of two reduced fat croissants with Buxton water (approximately $316 \mathrm{kcal}$ ). Following this, the cognitive and mood test battery was completed which took approximately an hour and a half to administer. The intervention was then consumed with the volunteer being asked to consume it as quickly as possible. One hour post-consumption, the BP measurements were repeated and a second blood sample taken, followed by the DVP measurements. The volunteer was then given a standardised low-polyphenol lunch which was consumed within 20 minutes and consisted of a white bread sandwich with light soft cheese, a packet of Walker's lights ready salted crisps and Buxton mineral water (approximately $429 \mathrm{kcal}$ in total). The cognitive test battery was then completed at two and five hours post consumption of the intervention. The volunteer was offered a snack which was in keeping with the lowpolyphenol diet (a reduced fat croissant) during the one and a half hour break between the two final test- ing sessions. See Fig. 1 for a schematic of the test day timeline.

All procedures were in accordance with the guidelines of the 1975 Declaration of Helsinki and approved by the University of Reading Research Ethics Committee. This trial was registered at clinicaltrials.gov, unique identifier: NCT01289860.

\subsection{Measures}

\subsubsection{Primary endpoint: Cognitive function}

The tasks comprising the battery were piloted to determine appropriate sensitivity with parallel versions created that were equivalent in task difficulty. The global cognitive function measure consisted of the primary dependant variable from 14 tasks assessing executive function and memory (Go-NoGo, Stroop, Digit Switch, Continuous Performance Task, Digit Symbol Substitution Test, Random Word Generation, Three-Word Sets Task, N-back, Letter memory, Location Task, immediate and delayed recall and recognition, see Appendix 1 of the supplementary material for task descriptions). Performance on each individual cognitive task was also analysed to determine any specific cognitive benefits as a result of blueberry supplementation. Computerised tasks were administered using E-prime (Psychology Software Tools, Inc., Sharpsburg, PA, USA) and Visual Basic 6.0 (Microsoft) software.

\subsubsection{Secondary endpoints: Vascular function and blood sampling}

\subsubsection{Blood pressure}

Three readings were taken using a standardised automatic Sphygmomanometer (OMRON MX2

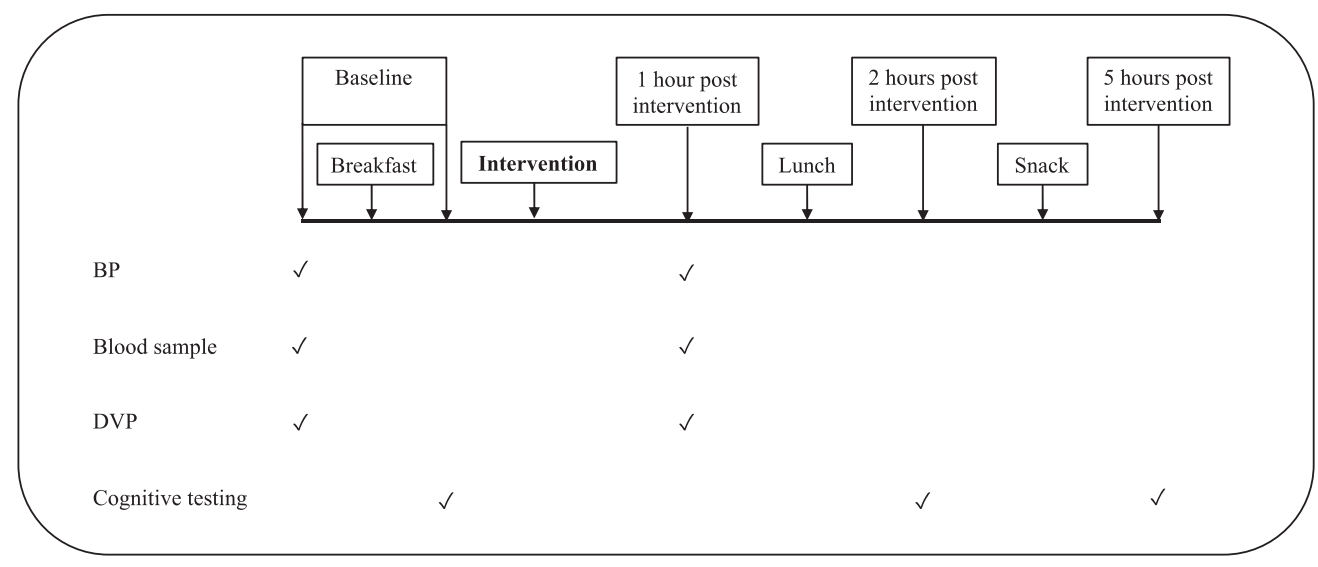

Fig. 1. Test day timeline. BP: Blood pressure; DVP: Digital Volume Pulse. 
automatic digital upper arms BP monitor, Milton Keynes, United Kingdom) and the average calculated. Measurements were taken on the left arm whilst the volunteer was resting in a seated position. Volunteers were asked to remain silent and not to cross their legs whilst the measurement was being taken.

\subsubsection{Stiffness index}

This is an estimate of large artery stiffness derived from a Digital Volume Pulse measurement (Micro Medical UK Ltd.) which is a non-invasive procedure. The volunteer sat in an upright position whilst a sensor was clipped onto their left thumb. They were asked to remain quiet, still and relaxed. Three successive readings were taken in order to obtain an average.

\subsubsection{Plasma concentration of BDNF}

Blood samples were taken via venepuncture by a trained Phlebotomist and drawn into a tube containing Ethylenediaminetetraacetic acid (EDTA). This was kept on ice until the sample could be processed (15-30 minutes post collection). Processing involved centrifuging the EDTA tube of whole blood at 3000 r.p.m, $4^{\circ} \mathrm{C}$ for $10-15$ minutes to separate the plasma which was subsequently aliquoted into vials each containing $500 \mathrm{~mL}$ and stored in $\mathrm{a}-20^{\circ} \mathrm{C}$ freezer for future analysis.

A Quantikine ${ }^{\circledR}$ human mature BDNF enzymelinked immunosorbent assay (ELISA) kit (R\&D systems, Inc.) was used to determine BDNF levels in plasma samples which were assayed in duplicate and according to the instructions of the manufacturer.

\subsection{Statistical procedures}

A composite measure of global cognitive function was calculated by converting the primary dependant variable from each task into $\mathrm{Z}$ scores ${ }^{2}$, combining these and dividing by the number of tasks contributing to the measure. Statistical analysis was then performed on this composite measure of global cognitive function ${ }^{3}$. SPSS version 21 (IBM, Armonk,

\footnotetext{
${ }^{2}$ Where appropriate, data were reverse scored so that a higher score indicted better performance. The grand mean and standard deviation for each task across all conditions was calculated; the grand mean was subtracted from each score and divided by the standard deviation.

${ }^{3}$ If a score for a particular task was missing for a session it was replaced with the mean score for that session. Analysis was also conducted with missing values and yielded comparable results (Control: $F(1,31)=4.09, \mathrm{p}=0.05)$.
}

NY, USA) was used to apply Linear Mixed Modelling to the data, employing an unstructured covariance matrix for repeated measurements. Intervention (Blueberry, Control), Time (2 hours, 5 hours) and Intervention * Time interaction were entered as fixed factors in the model, Participant ID as a random effect where possible (excluded if the validity of the results was compromised), and baseline cognitive function was included as a covariate. Bonferroni corrected pairwise comparisons were examined regardless of the significance of the overall $F$ test statistic (see Howell [35]). The data from the individual cognitive tasks were analysed by applying the same Linear Mixed Model to the primary dependant variable of each task.

For all physiological parameters (BP, stiffness index, plasma concentration of BDNF), change from baseline analysis was used to compare between interventions using paired sample $t$ tests.

\section{Results}

\subsection{Cognitive performance}

Whilst the overall Linear Mixed Model revealed that intervention was not a significant predictor of global cognitive performance (main effect: $p=0.81$; interaction: $p=0.18$ ), pairwise comparisons revealed significantly worse performance following the control drink at 2 compared to 5 hours post consumption (mean $=-0.06$ vs 0.06 respectively; $F(1,36)=4.60$, $p=0.04)$, whereas there was no significant difference in cognitive performance at 2 compared to 5 hours post consumption of the blueberry beverage $($ mean $=0.01$ vs 0.02 respectively; $F(1,36)=0.05$, $p=0.82$ ) (Fig. 2).

When cognitive tasks were analysed individually (Tables 4 and 5), for the digit switch task, analysis revealed a trend towards reduced switch cost following the blueberry (mean $=395.76)$ compared to the control $($ mean $=479.48)$ drink at the 2 hour timepoint $(F(1,15.8)=3.89, p=0.066)$, despite the overall intervention by time interaction failing to reach significance $(F(1,35)=0.518, p>0.05)$. For the random word generation task, performance declined over time $(F(1,35)=4.29, p=0.046)$, whereas for the three-word sets task, performance improved over time $(F(1,36)=17.54, p<0.01)$. However, there was no significant effect of the intervention or a time by intervention interaction for either of the tasks. For the immediate word recognition task, performance 


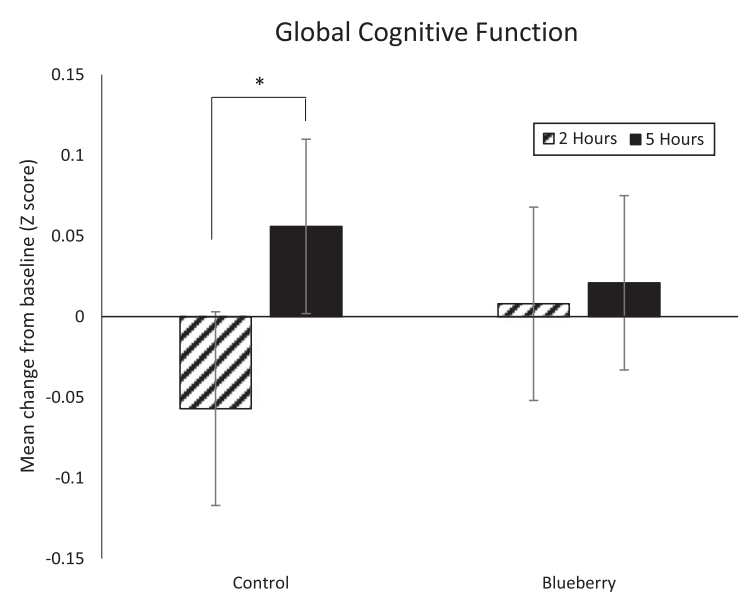

Fig. 2. Mean change in global cognitive function $( \pm$ SE) following control and blueberry interventions in relation to baseline. There was a significant decrease in performance in the control condition at 2 hours compared to at 5 hours $\left({ }^{*} p<0.05\right)$.

was better following the blueberry compared with the control intervention (mean $=26.71$ vs 25.81 respectively; $F(1,17.61)=4.29, p=0.05)$. Also, despite no significant intervention by time interaction, pairwise comparisons reveal significantly more words recognised following the blueberry compared to the control drink at the 2 hour (mean $=26.77$ vs 25.48 respectively; $F(1,16.30)=6.56, p=0.02)$ but not the 5 hour time point (mean $=26.65$ vs 26.15 respectively; $F(1,27.86)=0.60, p=0.44)$. Finally, despite an intervention by time interaction bordering on significance for the delayed word recognition $\operatorname{task}(F(1,34)=3.59$, $p=0.067)$, none of the pairwise comparisons were significant. There was no effect of the intervention for any of the other cognitive tasks.

\subsection{Vascular function and plasma concentration of BDNF}

As shown in Fig. 3, there was a trend towards the blueberry beverage attenuating the increase in systolic blood pressure evident following the control drink (mean change from baseline $=2.28$ vs 8.17 respectively; $t(17)=1.87, p=0.08 ; \mathrm{BCa} 95 \%$ CI $[-0.11,12.28])$. There was no statistically significant effect of intervention on stiffness index $(t(13)=-0.67, p>0.05 ;$ BCa 95\% CI $[-4.54,1.67])$, diastolic BP $(t(17)=0.89, p>0.05 ; \mathrm{BCa} 95 \% \mathrm{CI}$ $[-1.03,3.11])$, or pulse rate $(t(17)=0.12, p>0.05$; BCa 95\% CI $[-2.77,2.70])$. Also, despite a decline in mean plasma concentration of BDNF following the control beverage which appeared to be attenuated following the blueberry beverage (mean change from baseline $=-46.54$ vs -9.70 respectively), this difference was not significant $(t(8)=-1.79, p>0.05 ; \mathrm{BCa}$ $95 \%$ CI $\left.[-89.64,5.26]^{4}\right)$.

\section{Discussion}

Consumption of a beverage containing approximately $600 \mathrm{mg}$ of blueberry-derived flavonoids elicited a protective effect on global cognitive function in a sample of healthy older adults, sustaining cognitive performance across the day and protecting against the decline evident following the control beverage. These findings are in agreement with previous flavonoid intervention studies which have also found positive effects on global cognitive function in middle-aged and older adults [5, 6]. Furthermore, the trend towards the blueberry intervention attenuating a rise in systolic BP observed following the control intervention, suggests that the positive impact of flavonoids on vascular health could be a potential mechanism through which these natural compounds have beneficial effects on cognitive performance. Whilst the decrease in plasma concentration of BDNF following the control drink was attenuated following the blueberry drink, this was not significant and more data is required to determine whether BDNF and associated biochemical parameters may play a mechanistic role in cognitive benefits evident following consumption of flavonoid-rich interventions.

The analyses of the individual cognitive tasks suggest that the overall cognitive benefit may be driven by positive effects on episodic memory and executive function, namely recognition memory (immediate word recognition task) and switching ability (digit switch task) respectively. The memory-related findings are not only in agreement with acute memory effects reported in children $[11,12]$ but also with more recent studies on chronic supplementation such as those of Whyte et al. [24] who reported improvements on tests of episodic and spatial memory in older adults following chronic supplementation with a proprietary blueberry extract for 90 days. In addition, Bensalem et al. [25] report similar beneficial effects on episodic memory in terms of immediate free recall performance in a cohort of older adults following 6 months of supplementation with a polyphenolrich grape and blueberry extract, although here the

\footnotetext{
${ }^{4}$ Missing values imputed with mean of session as data was available for only a small sub-set of volunteers $(N=9)$ and to exclude cases with missing data would reduce this further. The result was the same when data were not imputed with $N=6(t(5)=-1.28$, $p>0.05 ; \mathrm{BCa} 95 \%$ CI $[-76.95,9.64])$
} 
Table 4

Data and results of the LMM analysis of executive function tasks; ${ }^{*} p<0.05 ;{ }^{* *} p<0.001$

\begin{tabular}{|c|c|c|c|c|c|c|c|c|}
\hline \multirow[t]{3}{*}{ Cognitive task } & & \multirow{3}{*}{$\begin{array}{l}\text { Estimated mean } \\
\text { (Standard error) }\end{array}$} & \multicolumn{2}{|c|}{ Main effect of time } & \multicolumn{2}{|c|}{ Main effect of condition } & \multirow{2}{*}{\multicolumn{2}{|c|}{$\frac{\text { Condition } * \text { time }}{\text { interaction }}$}} \\
\hline & & & \multirow[b]{2}{*}{$F$} & \multirow[b]{2}{*}{$\mathrm{p}$} & \multirow[b]{2}{*}{$\mathrm{F}$} & \multirow[b]{2}{*}{$\mathrm{p}$} & & \\
\hline & & & & & & & $\mathrm{F}$ & $\mathrm{p}$ \\
\hline \multicolumn{9}{|l|}{ Go-NoGo } \\
\hline Correct RT & & & 0.18 & 0.67 & 0.13 & 0.73 & 0.19 & 0.66 \\
\hline \multirow[t]{2}{*}{ Control } & 2 Hours & $627.52(9.79)$ & & & & & & \\
\hline & 5 Hours & $627.61(10.48)$ & & & & & & \\
\hline \multirow[t]{2}{*}{ Blueberry } & 2 Hours & $627.53(9.79)$ & & & & & & \\
\hline & 5 Hours & $620.49(10.48)$ & & & & & & \\
\hline Baseline covariate & & 618.64 & & & & & & \\
\hline \multicolumn{9}{|l|}{ Stroop } \\
\hline Correct RT & & & 0.05 & 0.83 & 1.91 & 0.19 & 0.06 & 0.81 \\
\hline \multirow[t]{2}{*}{ Control } & 2 Hours & $854.82(10.36)$ & & & & & & \\
\hline & 5 Hours & $855.04(10.82)$ & & & & & & \\
\hline \multirow[t]{2}{*}{ Blueberry } & 2 Hours & $869.37(10.36)$ & & & & & & \\
\hline & 5 Hours & $866.74(10.82)$ & & & & & & \\
\hline Baseline covariate & & 870.75 & & & & & & \\
\hline \multicolumn{9}{|l|}{ Digit Switch } \\
\hline Switch Cost & & & 0.93 & 0.34 & 2.41 & 0.15 & 0.52 & 0.48 \\
\hline \multirow[t]{2}{*}{ Control } & 2 Hours & $479.48(30.76)$ & & & & & & \\
\hline & 5 Hours & $485.70(39.35)$ & & & & & & \\
\hline \multirow[t]{2}{*}{ Blueberry } & 2 Hours & $395.76(31.63)$ & & & & & & \\
\hline & 5 Hours & $438.46(40.47)$ & & & & & & \\
\hline Baseline covariate & & 544.61 & & & & & & \\
\hline \multicolumn{9}{|l|}{ CPT } \\
\hline Commission Errors & & & 1.21 & 0.28 & 0.001 & 0.97 & 0.66 & 0.42 \\
\hline Control & 2 Hours & $7.83(1.32)$ & & & & & & \\
\hline & 5 Hours & $6.72(1.31)$ & & & & & & \\
\hline Blueberry & 2 Hours & $7.34(1.32)$ & & & & & & \\
\hline & 5 Hours & $7.17(1.31)$ & & & & & & \\
\hline Baseline covariate & & 8.50 & & & & & & \\
\hline DSST & & & & & & & & \\
\hline Total Correct & & & 0.09 & 0.76 & 0.004 & 0.95 & 0.82 & 0.37 \\
\hline Control & 2 Hours & $55.90(0.86)$ & & & & & & \\
\hline & 5 Hours & $55.57(1.05)$ & & & & & & \\
\hline Blueberry & 2 Hours & $55.49(0.86)$ & & & & & & \\
\hline & 5 Hours & $56.15(1.05)$ & & & & & & \\
\hline Baseline covariate & & 53.61 & & & & & & \\
\hline RWG & & & & & & & & \\
\hline Total Correct & & & 4.29 & $0.046^{*}$ & 0.39 & 0.55 & 0.06 & 0.81 \\
\hline Control & 2 Hours & $34.93(1.44)$ & & & & & & \\
\hline & 5 Hours & $33.40(1.33)$ & & & & & & \\
\hline Blueberry & 2 Hours & $34.48(1.41)$ & & & & & & \\
\hline & 5 Hours & $32.53(1.31)$ & & & & & & \\
\hline Baseline covariate & & 26.54 & & & & & & \\
\hline Three-Word Sets & & & & & & & & \\
\hline Total Correct & & & 17.54 & $<.001^{* *}$ & 0.59 & 0.45 & 0.60 & 0.44 \\
\hline Control & 2 Hours & $2.82(0.38)$ & & & & & & \\
\hline & 5 Hours & $4.59(0.36)$ & & & & & & \\
\hline Blueberry & 2 Hours & $2.80(0.38)$ & & & & & & \\
\hline & 5 Hours & $4.02(0.36)$ & & & & & & \\
\hline Baseline covariate & & 4.08 & & & & & & \\
\hline
\end{tabular}


Table 4

(Continued)

\begin{tabular}{|c|c|c|c|c|c|c|c|c|}
\hline \multirow[t]{3}{*}{ Cognitive task } & & \multirow{3}{*}{$\begin{array}{l}\text { Estimated mean } \\
\text { (Standard error) }\end{array}$} & \multicolumn{2}{|c|}{ Main effect of time } & \multicolumn{2}{|c|}{ Main effect of condition } & \multirow{2}{*}{\multicolumn{2}{|c|}{$\begin{array}{c}\text { Condition * time } \\
\text { interaction }\end{array}$}} \\
\hline & & & \multirow[b]{2}{*}{$F$} & & & & & \\
\hline & & & & $\mathrm{p}$ & $\mathrm{F}$ & $\mathrm{p}$ & $\mathrm{F}$ & $\mathrm{p}$ \\
\hline N-Back Percent correct & & & 0.31 & 0.58 & $<0.001$ & 0.998 & 0.01 & 0.93 \\
\hline Control & 2 Hours & $73.32(4.49)$ & & & & & & \\
\hline & 5 Hours & $74.76(4.42)$ & & & & & & \\
\hline Blueberry & 2 Hours & $73.03(4.49)$ & & & & & & \\
\hline & 5 Hours & $75.01(4.42)$ & & & & & & \\
\hline Baseline covariate & & 72.48 & & & & & & \\
\hline Letter Memory Total Correct & & & 0.05 & 0.83 & 1.57 & 0.22 & 0.19 & 0.67 \\
\hline Control & 2 Hours & $5.63(0.43)$ & & & & & & \\
\hline & 5 Hours & $5.79(0.38)$ & & & & & & \\
\hline Blueberry & 2 Hours & $5.10(0.43)$ & & & & & & \\
\hline & 5 Hours & $5.04(0.38)$ & & & & & & \\
\hline Baseline covariate & & 4.67 & & & & & & \\
\hline Location task Adjusted correct & & & 0.26 & 0.62 & 0.996 & 0.33 & 1.56 & 0.22 \\
\hline Control & 2 Hours & $-5.00(0.85)$ & & & & & & \\
\hline & 5 Hours & $-4.39(0.88)$ & & & & & & \\
\hline Blueberry & 2 Hours & $-3.11(0.85)$ & & & & & & \\
\hline & 5 Hours & $-4.56(0.88)$ & & & & & & \\
\hline Baseline covariate & & -5.19 & & & & & & \\
\hline
\end{tabular}

Table 5

Data and results of the LMM analysis of memory tasks; ${ }^{\wedge} p<0.07$

\begin{tabular}{|c|c|c|c|c|c|c|c|c|}
\hline \multirow[t]{3}{*}{ Cognitive task } & & \multirow{3}{*}{$\begin{array}{l}\text { Estimated mean } \\
\text { (Standard error) }\end{array}$} & \multicolumn{2}{|c|}{ Main effect of time } & \multicolumn{2}{|c|}{ Main effect of condition } & \multirow{2}{*}{\multicolumn{2}{|c|}{$\frac{\text { Condition } * \text { time }}{\text { interaction }}$}} \\
\hline & & & \multirow[b]{2}{*}{$F$} & \multirow[b]{2}{*}{$\mathrm{p}$} & \multirow[b]{2}{*}{$\mathrm{F}$} & \multirow[b]{2}{*}{$\mathrm{p}$} & & \\
\hline & & & & & & & $\mathrm{F}$ & $\mathrm{p}$ \\
\hline \multicolumn{9}{|l|}{ Immediate recall } \\
\hline Total correct & & & 0.32 & 0.57 & 0.28 & 0.61 & 1.76 & 0.19 \\
\hline \multirow[t]{2}{*}{ Control } & 2 Hours & $7.97(0.47)$ & & & & & & \\
\hline & 5 Hours & $7.41(0.46)$ & & & & & & \\
\hline \multirow[t]{2}{*}{ Blueberry } & 2 Hours & $7.37(0.47)$ & & & & & & \\
\hline & 5 Hours & $7.59(0.46)$ & & & & & & \\
\hline Baseline covariate & & 8.58 & & & & & & \\
\hline \multicolumn{9}{|l|}{ Delayed recall } \\
\hline Total correct & & & 1.12 & 0.30 & 0.43 & 0.52 & 0.003 & 0.95 \\
\hline \multirow[t]{2}{*}{ Control } & 2 Hours & $5.52(0.56)$ & & & & & & \\
\hline & 5 Hours & $5.92(0.68)$ & & & & & & \\
\hline \multirow[t]{2}{*}{ Blueberry } & 2 Hours & $5.07(0.56)$ & & & & & & \\
\hline & 5 Hours & $5.51(0.66)$ & & & & & & \\
\hline Baseline covariate & & 7.65 & & & & & & \\
\hline \multicolumn{9}{|c|}{ Immediate recognition } \\
\hline Total correct & & & 0.49 & 0.49 & 4.29 & $0.05^{\wedge}$ & 1.00 & 0.32 \\
\hline \multirow[t]{2}{*}{ Control } & 2 Hours & $25.48(0.51)$ & & & & & & \\
\hline & 5 Hours & $26.15(0.59)$ & & & & & & \\
\hline \multirow[t]{2}{*}{ Blueberry } & 2 Hours & $26.77(0.52)$ & & & & & & \\
\hline & 5 Hours & $26.65(0.60)$ & & & & & & \\
\hline Baseline covariate & & 26.80 & & & & & & \\
\hline \multicolumn{9}{|l|}{ Delayed recognition } \\
\hline Total correct & & & 0.20 & 0.66 & 0.03 & 0.86 & 3.59 & $0.067^{\wedge}$ \\
\hline \multirow[t]{2}{*}{ Control } & 2 Hours & $22.00(0.85)$ & & & & & & \\
\hline & 5 Hours & $22.89(1.01)$ & & & & & & \\
\hline \multirow[t]{2}{*}{ Blueberry } & 2 Hours & $22.94(0.90)$ & & & & & & \\
\hline & 5 Hours & $21.51(1.07)$ & & & & & & \\
\hline Baseline covariate & & 24.12 & & & & & & \\
\hline
\end{tabular}




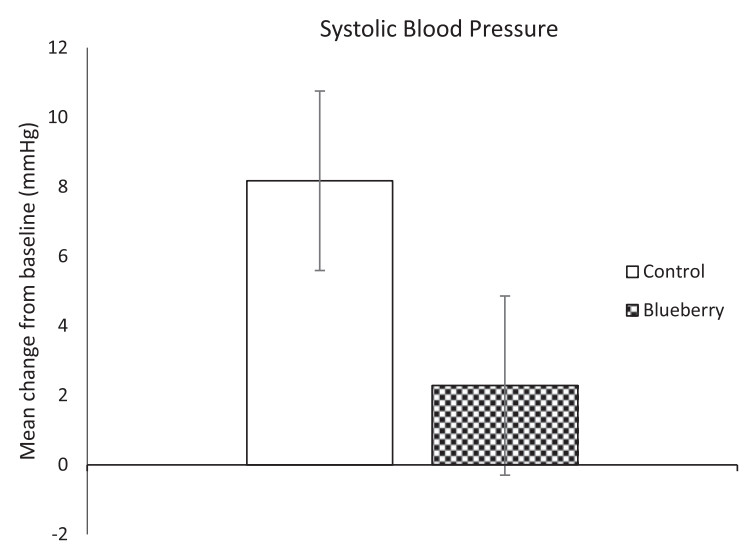

Fig. 3. Change in systolic blood pressure following control and blueberry interventions in relation to baseline.

greatest memory benefits were evident in the most cognitively impaired subjects.

Despite increasing evidence to suggest that cognitive benefits in older adults following acute flavonoid supplementation are more likely to be in the form of improvements to memory [1], the positive effects of a blueberry beverage on a test of executive ability in the current study supports research such as that of Miller et al. [36], who report that older adults improved on measures of executive function following blueberry supplementation compared to those in the placebo group. However, this was after chronic intake over a similar time frame to the study by Whyte et al. [24]. Taken together, this suggests that in addition to the benefits to episodic memory evident following flavonoid supplementation, it is also possible to see improvement in other cognitive domains.

Whilst there was no significant effect of the intervention for the other cognitive tasks in the test battery, studies such as that of Boespflug et al. [37] have demonstrated physiological effects of flavonoids on the brain in older adults with mild cognitive impairment, in the absence of a behavioural effect for a specific cognitive task, which could explain the non-significant behavioural findings for some of the tasks in the current study. In addition, Igwe et al. [38] supplemented younger and older adults with anthocyanin-rich plum juice and found benefits to vascular parameters such as reduced blood pressure, but no cognitive effects. Therefore, compared to physiological effects, behavioural benefits to cognitive performance following flavonoid supplementation may be more subtle and difficult to detect. As such, it is essential that future research efforts should continue to optimise the sensitivity of cognitive measures.
Also worth discussion is the finding that performance was greater at the 5 hour time point compared to at 2 hours following the control intervention. It would be reasonable to hypothesise that performance would likely decline over the duration of the visit due to fatigue and as such to expect worse rather than better performance at the 5 hour timepoint. However, the long break between the two post drink testing periods could account for why performance was not worse at the five hour timepoint following both interventions. Furthermore, the effect of practice should also be considered. However, there was no significant main effect of time for global cognitive function; it was not the case that performance at 5 hours post consumption was significantly greater than at 2 hours regardless of intervention. This is reflected in the fact that even though performance was better 5 hours post consumption of the blueberry intervention compared to at the 2 hour timepoint, this difference was not significant. It is therefore unlikely that better performance at the 5 hour timepoint following the control drink is due to practice alone. An alternative explanation is that performance at the 2 hour timepoint is affected by the 'post lunch dip' and that the blueberry beverage protects against the decline in cognitive performance that can be evident following a meal [39].

For both interventions, cognitive performance was best at 5 hours post consumption of the drinks and whilst this appeared to be greater following the control compared to the blueberry drink, this difference was not significant. Also, whilst practice may have contributed to greater improvement in cognitive function at the 5 hour timepoint, as mentioned previously, there was no significant main effect of time and other possible explanations should be considered. Several flavonoid intervention studies have demonstrated improved cognitive performance at around 6 hours post consumption (see Bell et al. [1]) which could be due to delayed release of metabolites and catabolites following extensive digestion and metabolism of flavonoids in the gut [40]. It is likely to be these metabolites which are responsible for health benefits [41] such as improved vascular function where increases in FMD response have been demonstrated six hours post consumption of blueberry [30], and could include cognitive effects. However, in the present study, performance was not significantly better following the blueberry compared to the control drink at either post drink timepoint. One possible explanation is that with increasing age, the number of beneficial microorganisms in the colon decrease [42], which is likely to include those involved in flavonoid metabolism, and could potentially explain any delay 
in absorption in the current study considering the population tested. Furthermore, the intervention powders were suspended in milk and whilst some research such as that of Draijer et al. [43] suggests that dairy proteins do not affect the bioavailability of polyphenols, there is also evidence to the contrary, with a study by Xiao et al. [44] reporting that consuming milk with anthocyanins can affect their absorption. Overall, this suggests that the 5 hour post drink timepoint in the current study could have been too early to capture the maximum cognitive benefit that may otherwise have been evident following the acute anthocyanin-rich blueberry intervention.

The blueberry beverage was also found to attenuate the increase in systolic blood pressure which was evident following consumption of the control drink, although this was not statistically significant. In addition, diastolic blood pressure decreased following the blueberry drink but this was not significantly greater than the reduction seen post consumption of the control drink. Comparable vascular effects following flavonoid supplementation in older adults have been documented elsewhere, with Whyte et al. [24] recently reporting systolic blood pressure lowering effects of a wild blueberry extract compared to placebo at both 3 and 6 months of daily supplementation. In addition, Barona et al. [45] also report reduced systolic but not diastolic BP following 30 day supplementation with grape polyphenols in individuals with metabolic syndrome. Blood pressure lowering effects have also been found following blueberry supplementation for 8 weeks in post menopausal women with pre- or stage- 1 hypertension [46] as well as in individuals with metabolic syndrome [47]. Taken together, these findings support those of the current trial and berry-derived flavonoids may have beneficial effects on blood pressure in both healthy and at-risk populations.

Acute effects of flavonoids on blood pressure have also been investigated. In a study by Kent et al. [48], a single $300 \mathrm{~mL}$ dose of anthocyanin-rich cherry juice has been shown to lower blood pressure 2 hours post consumption, which could explain why there was a trend rather than a significant effect in the current study, as the timepoint at which the BP measures were taken may have been too early to capture the optimum benefit. Also, whilst the cause of the increase in systolic blood pressure following the control intervention in the current study is unclear, it could be related to anxiety experienced in anticipation of repeated measurements which was attenuated following the blueberry intervention. Anxiolytic effects have been observed following flavonoid supplemen- tation in rats [49] and humans [50], suggesting that such indirect effects should be considered in addition to any mechanistic vascular rationale behind blood pressure lowering effects of berries.

Supporting healthy vascular function is likely to translate to cerebrovascular benefits and improving cerebral blood flow has been proposed as a potential explanation for the beneficial effects of flavonoids on cognitive function [19]. Several human intervention studies have reported increases in cerebral blood flow or volume following acute cocoa flavanol supplementation in both younger and older adults $[16$, 17-51], whilst Bowtell et al. [15] found increased perfusion and activation in brain regions associated with cognitive function following 12 weeks of blueberry supplementation in healthy older adults. However, a limitation of the current study is that cerebral blood flow was not directly measured and therefore assertions that this could be a potential explanation for the protective effects of the blueberry beverage on cognitive performance should be made with caution. Further research into the effects of flavonoid supplementation on cognitive function, endothelial function and cerebral blood flow is therefore required to test this theory.

Brain-derived neurotrophic factor is a neurotrophin which is involved in activity-dependent synaptic plasticity and plays an important role in learning and memory [52]. Flavonoid-induced increases in circulating BDNF is therefore another potential mechanism of action and in the present study the blueberry beverage appeared to attenuate the decline in plasma concentration seen following consumption of the control beverage, albeit not significantly. BDNF concentration can reduce during the day in men [53] which could be a contributing factor to the reduction in concentration seen post consumption of the control beverage. Seeing as this protein is associated with memory and learning, the relative maintenance of plasma concentration of BDNF following the blueberry beverage is therefore a physiological benefit. Favourable effects have also been reported elsewhere, with studies in rodents demonstrating flavonoid induced increases in BDNF in the hippocampus, an area of the brain associated with memory processes, following blueberry supplementation [20-54]. Furthermore, a recent human intervention study reported increased serum levels of BDNF following flavonoid supplementation which were correlated with improved global cognitive function [55].

Whilst the results are promising, there were no significant effects on stiffness index, diastolic blood 
pressure or pulse rate. Stiffness index may be more likely to change following chronic flavonoid intervention and a more sensitive measure of vascular reactivity such as FMD (flow mediated dilatation) may be more appropriate for acute supplementation. Indeed, Rodriguez-Mateos et al. have shown that acute supplementation with blueberry [30] and cranberry-derived flavonoids [56] in healthy men resulted in increased FMD response which was associated with plasma concentration of flavonoid metabolites. Also, Whyte et al. [24] have recently reported an isolated effect of a blueberry intervention on systolic blood pressure and no effect on any other vascular measures in older adults. The reason for this is currently unclear but may be related to the impact of flavonoids on cell signalling pathways associated with specific vascular outcomes. Benefits to peripheral vascular health are important as this could result in increased cerebral blood flow, for example through improved vascular reactivity, which has implications for brain and cognitive function [57].

A further limitation of the current research is the small sample size, as well as large inter-individual variability in the data. A lack of statistical power may therefore partly explain the non-significant results if the effects were too subtle to be detected in such a relatively small and heterogenous sample. Finally, the lack of bioavailability data means it is not possible to directly associate flavonoid intake with the benefits to cognitive function, blood pressure and plasma concentration of BDNF reported herein. However, it is noteworthy that the protective effect of the blueberry intervention on cognitive performance was at the 2 hour time point, which is compatible with research that has reported circulating flavonoid metabolites at 1-2 hours post consumption of a blueberry intervention [30], as behavioural effects could be delayed. Considering that cognitive function was not measured an hour post consumption of the intervention, it is unclear whether maximal cognitive benefit may have been missed. Despite the limitations of this study, the data are promising and merit further research.

In conclusion, consumption of flavonoid-rich blueberries could be beneficial in terms of maintaining cognitive function in a healthy older adult population. Benefits could be due to the interaction of berry-derived flavonoids with cell signalling pathways associated with cognitive performance and helping to maintain healthy cerebrovascular function. Initial evidence from human intervention trials of flavonoid supplementation in this population
[6-36-58] as well as individuals with mild cognitive impairment [22, 23-37] is promising. However, further research combining the latest brain imaging techniques with biomarkers of cognitive and vascular function, bioavailability of flavonoid metabolites and behavioural testing, would be valuable in improving our understanding of the possible mechanisms through which flavonoid-rich foods may have the potential to promote healthy cognitive ageing by slowing age-related cognitive decline and the onset of neurodegenerative disease.

\section{Acknowledgments}

This research was completed as part of a PhD studentship funded by the Graduate School of Social Sciences at the University of Reading. The authors would also like to thank Ana Rodriguez-Mateos for her assistance with the polyphenol analysis of the blueberry intervention, as well as the volunteers who took part in the study without whom it would not have been possible.

\section{Funding}

The authors report no funding.

\section{Conflict of interest}

The authors have no conflict of interest to report.

\section{Supplementary Material}

The appendix is available in the electronic version of this article: http://dx.doi.org/10.3233/NHA180056.

\section{References}

[1] Bell L, Lamport DJ, Butler LT, Williams CM. A review of the cognitive effects observed in humans following acute supplementation with flavonoids, and their associated mechanisms of action. Nutrients. 2015;7:10290-306.

[2] Lamport DJ, Dye L, Wightman JD, Lawton CL. The effects of flavonoid and other polyphenol consumption on cognitive performance: A systematic research review of human experimental and epidemiological studies. Nutrition and Ageing. 2012;1:5-25. 
[3] Macready AL, Kennedy OB, Ellis JA, Williams CM, Spencer JPE, Butler LT. Flavonoids and cognitive function: a review of human randomized controlled trial studies and recommendations for future studies. Genes Nutrition. 2009;4:227-42.

[4] Scholey AB, French SJ, Morris PJ, Kennedy DO, Milne $\mathrm{AL}$, Haskell CF. Consumption of cocoa flavanols results in acute improvements in mood and cognitive performance during sustained mental effort. Psychopharmacology. 2010;24(10):1505-14.

[5] Alharbi M, Lamport D, Dodd G, Saunders C, Harkness $\mathrm{L}$, Butler L, et al. Flavonoid-rich orange juice is associated with acute improvements in cognitive function in healthy middle-aged males. Eur J Nutr. 2015 2015/08/18:1-9. English.

[6] Kean RJ, Lamport DJ, Dodd GF, Freeman JE, Williams $\mathrm{CM}$, Ellis JA, et al. Chronic consumption of flavanonerich orange juice is associated with cognitive benefits: an 8-wk, randomized, double-blind, placebo-controlled trial in healthy older adults. American Journal of Clinical Nutrition. 2015;101(3):506-14.

[7] Nilsson A, Salo I, Plaza M, Bjorck I. Effects of a mixed berry beverage on cognitive functions and cardiometabolic risk markers; A randomized cross-over study in healthy older adults. PLoS One. 2017;12(11):e0188173. PubMed PMID: 29141041. PMCID: PMC5687726. Epub 2017/11/16.

[8] Watson AW, Haskell-Ramsay CF, Kennedy DO, Cooney JM, Trower T, Scheepens A. Acute supplementation with blackcurrant extracts modulates cognitive functioning and inhibits monoamine oxidase-B in healthy young adults. Journal of Functional Foods. 2015;17:524-39.

[9] Haskell-Ramsay CF, Stuart RC, Okello EJ, Watson AW. Cognitive and mood improvements following acute supplementation with purple grape juice in healthy young adults. Eur J Nutr. 2017 Dec;56(8):2621-31. PubMed PMID: 28429081. PMCID: PMC5682870. Epub 2017/04/22.

[10] Hosseinian FS, Beta T. Saskatoon and Wild Blueberries Have Higher Anthocyanin Contents than Other Manitoba Berries. Journal of Agricultural and Food Chemistry. 2007;55:10832-8.

[11] Whyte AR, Williams CM. Effects of a single dose of a flavonoid-rich blueberry drink on memory in 8 to $10 \mathrm{y}$ old children. Nutrition. 2015;31(3):531-4.

[12] Whyte AR, Schafer G, Williams CM. Cognitive effects following acute wild blueberry supplementation in 7- to 10-year-old children. Eur J Nutr. 2015 Sep;55(6):2151-62. PubMed PMID: 26437830. Epub 2015/10/07.

[13] Shukitt-Hale B, Carey AN, Jenkins D, Rabin BM, Joseph JA. Beneficial effects of fruit extracts on neuronal function and behavior in a rodent model of accelerated aging. Neurobiology of Aging. 2007;28(8):1187-94.

[14] Shukitt-Hale B, Galli RL, Meterko V, Carey A, Bielinski DF, McGhie T, et al. Dietary supplementation with fruit polyphenolics ameliorates age-related deficits in behavior and neuronal markers of inflammation and oxidative stress. Age. 2005;27:49-57.

[15] Bowtell JL, Aboo-Bakkar Z, Conway ME, Adlam ALR, Fulford J. Enhanced task-related brain activation and resting perfusion in healthy older adults after chronic blueberry supplementation. Applied Physiology, Nutrition, and Metabolism. 2017 2017/07/01;42(7):773-9.

[16] Brickman AM, Khan UA, Provenzano FA, Yeung L-K, Suzuki W, Schroeter H, et al. Enhancing dentate gyrus func- tion with dietary flavanols improves cognition in older adults Nature Neuroscience. 2014;17(12):1798-803.

[17] Francis ST, Head K, Morris PG, Macdonald IA. The effect of flavanol-rich cocoa on the fMRI response to a cognitive task in healthy young people. Journal of Cardiovascular Pharmacology. 2006;47:S215-S20. PubMed PMID: ISI:000239144600018.

[18] Sorond FA, Lipsitz LA, Hollenberg NK, Fisher NDL. Cerebral blood flow response to flavanol-rich cocoa in healthy elderly humans. Neuropsychiatric Disease and Treatment. 2008;4(2):433-40.

[19] Spencer JPE, Vauzour D, Rendeiro C. Flavonoids and cognition: The molecular mechanisms underlying their behavioural effects. Archives of Biochemistry and Biophysics. 2009;492:1-9.

[20] Rendeiro C, Vauzour D, Kean R, Butler L, Rattray M, Spencer JE, et al. Blueberry supplementation induces spatial memory improvements and region-specific regulation of hippocampal BDNF mRNA expression in young rats. Psychopharmacology. 2012 2012/10/01;223(3):319-30. English.

[21] Krikorian R, Shidler MD, Nash TA, Kalt W, VinqvistTymchuk MR, Shukitt-Hale B, et al. Blueberry Supplementation Improves Memory in Older Adults Journal of Agricultural and Food Chemistry. 2010;58:3996-4000.

[22] Desideri G, Kwik-Uribe C, Grassi D, Necozione S, Ghiadoni L, Mastroiacovo D, et al. Benefits in Cognitive Function, Blood Pressure, and Insulin Resistance Through Cocoa Flavanol Consumption in Elderly Subjects With Mild Cognitive Impairment. The Cocoa, Cognition, and Aging (CoCoA) Study. Hypertension. 2012;60:794-801.

[23] Krikorian R, Nash TA, Shidler MD, Shukitt-Hale B, Joseph JA. Concord grape juice supplementation improves memory function in older adults with mild cognitive impairment. British Journal of Nutrition. 2010;103:730-4.

[24] Whyte AR, Cheng N, Fromentin E, Williams CM. A Randomized, Double-Blinded, Placebo-Controlled Study to Compare the Safety and Efficacy of Low Dose Enhanced Wild Blueberry Powder and Wild Blueberry Extract (ThinkBlue) in Maintenance of Episodic and Working Memory in Older Adults. Nutrients. 2018 May 23;10(6). PubMed PMID: 29882843. PMCID: PMC6024810.

[25] Bensalem J, Dudonne S, Etchamendy N, Pellay H, Amadieu $\mathrm{C}$, Gaudout D, et al. Polyphenols from grape and blueberry improve episodic memory in healthy elderly with lower level of memory performance: a bicentric double-blind, randomized, placebo-controlled clinical study. J Gerontol A Biol Sci Med Sci. 2018 Jul 19. PubMed PMID: 30032176.

[26] Faul F, Erdfelder E, Lang A-G, Buchner A. G*Power 3: A flexible statistical power analysis program for the social, behavioral, and biomedical sciences. Behavior Research Methods. 2007;39:175-91.

[27] How PS, Ellis JA, Spencer JPE, Williams C. The impact of plant-derived flavonoids on mood, memory and motor skills in UK adults. Appetite. 2008;51(3):754.

[28] Haskell-Ramsay CF, Stuart RC, Okello EJ, Watson AW. Cognitive and mood improvements following acute supplementation with purple grape juice in healthy young adults. Eur J Nutr. 2017;56:2621-31.

[29] NHS. 5 A Day portion sizes 2018.

[30] Rodriguez-Mateos A, Rendeiro C, Bergillos-Meca T, Tabatabaee S, George TW, Heiss C, et al. Intake and time 
dependence of blueberry flavonoid-induced improvements in vascular function: a randomized, controlled, double-blind, crossover intervention study with mechanistic insights into biological activity. The American Journal of Clinical Nutrition. 2013 November 1, 2013;98(5):1179-91.

[31] Derogatis LR, Melisaratos N. The Brief Symptom Inventory: an introductory report. Psychological Medicine. 1983;13(3):595-605.

[32] Folstein MF, Folstein SE, McHugh PR. Mini Mental State: A practical method for grading the cognitive state of patients for the clinician. Journal of Psychiatric Research. 1975;12:18998.

[33] Cattell RB. Culture Fair Intelligence Test: A measure of "g". Savoy, IL: Institute for Personality and Ability Testing Inc. 1950.

[34] Nelson HE. The National Adult Reading Test (NART): test manual. NFER- Nelson. 1982.

[35] Howell DC. Statistical methods for psychology: Wadsworth Cengage Learning; 2013.

[36] Miller MG, Hamilton DA, Joseph JA, Shukitt-Hale B. Dietary blueberry improves cognition among older adults in a randomized, double-blind, placebo-controlled trial. Eur J Nutr. 2018;57:1169-80.

[37] Boespflug EL, Eliassen JC, Dudley JA, Shidler MD, Kalt W, Summer SS, et al. Enhanced neural activation with blueberry supplementation in mild cognitive impairment. Nutritional Neuroscience. 2017:1-9.

[38] Igwe EO, Charlton KE, Roodenrys S, Kent K, Fanning K, Netzel ME. Anthocyanin-rich plum juice reduces ambulatory blood pressure but not acute cognitive function in younger and older adults: a pilot crossover dose-timing study. Nutrition Research. 2017;47:28-43.

[39] Monk TH. The Post-Lunch Dip in Performance. Clinics in Sports Medicine. 2005;24(2):e15-e23.

[40] Williamson G, Clifford MN. Role of the small intestine, colon and microbiota in determining the metabolic fate of polyphenols. Biochemical Pharmacology. 2017;139:24-39.

[41] Moco S, Martin F-PJ, Rezzi S. Metabolomics View on Gut Microbiome Modulation by Polyphenol-rich Foods. Journal of Proteome Research. 2012 2012/10/05;11(10):4781-90.

[42] Woodmansey EJ. Intestinal bacteria and ageing. Journal of Applied Microbiology. 2007;102(5):1178-86.

[43] Draijer R, van Dorsten FA, Zebregs YE, Hollebrands B, Peters S, Duchateau GS, et al. Impact of Proteins on the Uptake, Distribution, and Excretion of Phenolics in the Human Body. Nutrients. 2016;8(12):814.

[44] Xiao D, Sandhu A, Huang YC, Park E, Edirisinghe I, Burton-Freeman BM. The effect of dietary factors on strawberry anthocyanins oral bioavailability. Food \& Function. 2017;8(11):3970-9.

[45] Barona J, Aristizabal JC, Blesso CN, Volek JS, Fernandez ML. Grape Polyphenols Reduce Blood Pressure and Increase Flow-Mediated Vasodilation in Men with Metabolic Syndrome. The Journal of Nutrition. 2012;142(9): 1626-32.

[46] Johnson SA, Figueroa A, Navaei N, Wong A, Kalfon R, Ormsbee LT, et al. Daily Blueberry Consumption Improves Blood Pressure and Arterial Stiffness in Postmenopausal Women with Pre- and Stage 1-Hypertension: A Randomized, Double-Blind, Placebo-Controlled Clinical Trial. Journal of the Academy of Nutrition and Dietetics. 2015;115(3):36977.
[47] Basu A, Du M, Leyva MJ, Sanchez K, Betts NM, Wu M, et al. Blueberries Decrease Cardiovascular Risk Factors in Obese Men and Women with Metabolic Syndrome. The Journal of Nutrition. 2010;140(9):1582-7.

[48] Kent K, Charlton KE, Jenner A, Roodenrys S. Acute reduction in blood pressure following consumption of anthocyanin-rich cherry juice may be dose-interval dependant: a pilot cross-over study. International Journal of Food Sciences and Nutrition. 2016;67(1):47-52.

[49] Tomic M, Ignjatovic D, Tovilovic-Kovacevic G, KrsticMilosevic D, Rankovic S, Popovic T, et al. Reduction of anxiety-like and depression-like behaviors in rats after one month of drinking Aronia melanocarpa berry juice. Food \& Function. 2016;7(7):3111-20.

[50] Watson AW, Okello EJ, Brooker HJ, Lester S, McDougall GJ, Wesnes KA. The impact of blackcurrant juice on attention, mood and brain wave spectral activity in young healthy volunteers. Nutr Neurosci. 2018 Jan 17:1-11. PubMed PMID: 29343201. Epub 2018/01/19.

[51] Lamport DJ, Pal D, Moutsiana C, Field DT, Williams CM, Spencer JPE, et al. The effect of flavanol-rich cocoa on cerebral perfusion in healthy older adults during conscious resting state: a placebo controlled, crossover, acute trial. Psychopharmacology. 2015;232(17):3227-34.

[52] Yamada K, Nabeshima T. Brain-Derived Neurotrophic Factor/TrkB Signaling in Memory Processes. Journal of Pharmacological Sciences. 2003;91:267-70.

[53] Choi SW, Bhang S, Ahn JH. Diurnal variation and gender differences of plasma brain-derived neurotrophic factor in healthy human subjects. Psychiatry Res. 2011 Apr 30;186(2-3):427-30. PubMed PMID: 20826006. Epub 2010/ $09 / 10$.

[54] Williams CM, El Mohsen MA, Vauzour D, Rendeiro C, Butler LT, Ellis JA, et al. Blueberry-induced changes in spatial working memory correlate with changes in hippocampal CREB phosphorylation and BDNF levels. Free Radical Biology and Medicine. 2008.

[55] Neshatdoust S, Saunders C, Castle SM, Vauzour D, Williams C, Butler L, et al. High-flavonoid intake induces cognitive improvements linked to changes in serum brain-derived neurotrophic factor: Two randomised, controlled trials. Nutr Healthy Aging. 2016;4:81-93.

[56] Rodriguez-Mateos A, Feliciano RP, Boeres A, Weber T, Nunes dos Santos C, Ventura MR, et al. Cranberry (poly)phenol metabolites correlate with improvements in vascular function: A double-blind, randomized, controlled, dose-response, crossover study. Molecular Nutrition \& Food Research. 2016;00:1-11.

[57] Williams RJ, Spencer JPE. Flavonoids, cognition, and dementia: Actions, mechanisms, and potential therapeutic utility for Alzheimer disease. Free Radical Biology \& Medicine. 2012;52:35-45.

[58] Mastroiacovo D, Kwik-Uribe C, Grassi D, Necozione S, Raffaele A, Pistacchio L, et al. Cocoa flavanol consumption improves cognitive function, blood pressure control, and metabolic profile in elderly subjects: the Cocoa, Cognition, and Aging (CoCoA) Study - a randomized controlled trial. American Journal of Clinical Nutrition. 2015;101:538-48. 